\title{
Prevalence of Non-Communicable Diseases in Schedule Caste dominated Rural Areas of Bhopal District, Madhya Pradesh
}

\author{
Yasmin Fatima', Abrar Mohd Khan ${ }^{2}$, Afshan Qaiser ${ }^{3}$, Amir Faisal Khan ${ }^{4}$, Tamanna Nazli \\ Abdul Raheem $^{6}$ \\ ${ }^{1,2}$ Research Associate (Unani), 3,4Research Officer, Clinical Research Unit Bhopal, Under CCRUM, Ministry of Ayush Govt. of India. \\ ${ }^{5,6}$ Research Officer, Central Council for Research in Unani Medicine (CCRUM), Ministry of Ayush Govt. of India. \\ DOI: https://doi.org/10.24321/2349.7181.202108
}

I $\quad \mathbf{N} \quad \mathbf{F}$

Corresponding Author:

Abrar Mohd Khan, Clinical Research Unit Bhopal, Under CCRUM, Ministry of Ayush Govt. of India. E-mail Id:

abrar.nium@gmail.com

Orcid Id:

https://orcid.org/0000-0001-6094-9275

How to cite this article:

Fatima Y, Khan AM, Qaiser A, Khan AF, Nazli T, Raheem A. Prevalence of Non-Communicable Diseases in Schedule Caste dominated Rural Areas of Bhopal District, Madhya Pradesh. J Adv Res Med. 2021;8(2):11-15.

Date of Submission: 2021-05-29

Date of Acceptance: 2021-06-17
$\begin{array}{llllllll}\mathbf{A} & \mathbf{B} & \mathbf{S} & \mathbf{T} & \mathbf{R} & \mathbf{A} & \mathbf{C} & \mathbf{T}\end{array}$

Introduction: Non-communicable diseases (NCDs) are the result of a combination of genetic, physiological, environmental and behavioral factors. Forty-one million people die each year due to NCDs, which is equivalent to $71 \%$ of all deaths globally.

Objective: The study was done to estimate the prevalence of noncommunicable diseases among five villages in the rural area of Bhopal, Madhya Pradesh, India.

Methodology: The present study was an observational study carried out on 6516 patients visiting mobile healthcare OPD conducted under Schedule Caste Sub-Plan in schedule caste dominated villages (Kurana, Kalkheda, Bagoniya, Toomda and Dhamarra) of Bhopal, Madhya Pradesh.

Results: It was found that $49.82 \%$ of the people from these villages were suffering from non-communicable diseases.

Conclusion: Nearly half of the population of this rural area was suffering from NCDs, therefore, people of rural areas are equally vulnerable to have NCDs. Urbanisation of lifestyle in rural population, poor education level and low economic status were the contributing factors.

Keywords: Non-Communicable Diseases (NCDs), CCRUM, Bhopal, Rural Area

\section{Introduction}

Non-communicable diseases (NCDs), better known as chronic diseases, tend to be of long duration. Some common NCDs are cardiovascular diseases (heart attacks and stroke), cancers, chronic respiratory diseases (such as chronic obstructive pulmonary disease, asthma) and diabetes. These are the result of a combination of genetic, physiological, environmental and behavioural factors. NCDs disproportionately affect people in low- and middle-income countries where more than three-quarters of global NCD deaths occur. ${ }^{1}$ The rise of NCDs has been driven primarily by four major risk factors: tobacco use, unhealthy dietary patterns, excessive use of alcohol and physical inactivity. ${ }^{2}$ People of all age groups, regions and countries are affected 
by NCDs. These conditions are often associated with older age groups, but evidence shows that 15 million of all deaths attributed to NCDs occur between the ages of 30 and 69 years. Of these "premature" deaths, over $85 \%$ are estimated to occur in low- and middle-income countries. Children, adults and the elderly all are vulnerable to the risk factors contributing to NCDs unhealthy diets, physical inactivity, exposure to tobacco smoke and the harmful use of alcohol. ${ }^{1,2}$

Unplanned urbanisation and globalisation is a major cause of unhealthy lifestyle. Lack of physical activity and an unhealthy diet may lead to hypertension, hyperglycaemia, hyperlipidemia and obesity, which can further lead to cardiovascular diseases. Many researchers have stated a close relationship between poverty and NCDs. Poor people are at a greater risk of being exposed to harmful products, such as tobacco, alcohol, smoking or unhealthy dietary practices, and have limited access to health services leading to death, as compared to rich people. ${ }^{1,2}$ Many researchers stated that heart disease, stroke, cancer, chronic respiratory diseases and diabetes are the main stream causes of mortality globally. Economic developments of many countries are disrupted due to such diseases. ${ }^{3}$

India is a developing country and many states in India are going through high rates of urbanisation. Urbanisation has led to economic improvement, but it has also resulted in some unhealthy habits such as increased junk food consumption, tobacco use, inappropriate lifestyle and decreased physical activity. ${ }^{4}$ NCDs are a major public health problem in India, especially cardiovascular diseases, diabetes mellitus, and stroke. NCDs are pose a serious challenges to Indian society and the economy. ${ }^{5,6}$ Unhealthy diet, physical inactivity and obesity are the common risk factors of NCDs. Policies and programmes focusing on reducing the load of these common risk factors are likely to make a significant impact on mitigating the mortality and morbidity due to these. ${ }^{7,8}$

Much attention has been paid to infectious diseases in lowand middle-income countries (LMICs) due to the significant morbidity and mortality arising from these diseases in endemic areas, yet the burden of non-communicable, chronic diseases is rising, ${ }^{9,10}$ accounting for approximately $50 \%$ of deaths in high-mortality regions of the world. ${ }^{11}$

Many researchers and agencies possibly assumed these diseases affect only the people from the upper class, but an increasing prevalence of this NCDs is low and middle class societies is major issue. The increase burden of these NCDs in villages are due to global changes in lifestyle, risk factors as well as changes in daily work activity, inappropriate transport facility, and reduced physical activity leading to a rise in unhealthy behaviors in these countries. ${ }^{10}$
NCDs are diseases that are not transmissible from one person to another. Main contributing factors are older age and unhealthy lifestyle-related behaviours, hence the term "lifestyle-related diseases." ${ }^{2}$

\section{Objective}

The study was done to estimate the prevalence of noncommunicable diseases among five villages in the rural area of Bhopal, Madhya Pradesh, India.

\section{Methodology}

The present study was an observational study under the Schedule Caste Sub-Plan carried out by clinical research unit Bhopal, one of the centres of Central Council for Research in Unani Medicine, an apex autonomous research organisation, functioning under the Ministry of Ayush, Govt. of India. Mobile Healthcare programme has been conducted amongst the SC population of five villages namely Kurana, Kalkheda, Bagoniya, Toomda and Dhamarra to provide free Unani treatment at their doorstep and also to create awareness among them. The data was collected in a predesigned validated format specially designed for Schedule Caste Sub-Plan during the period of July 2019 to March 2020 from the patients visiting mobile healthcare OPD. A total of 163 visits were made during the period and 6516 patients were treated under this program. Data collected were assessed to discover the prevalence of NCDs.

\section{Result \& Discussion}

The demographic profiles of the patients are mentioned in Table 1. A total of 6516 individuals were treated out of which 3042 (46.7\%) were male and 3474(53.3\%) were female. The majority $(5017,76.99 \%)$ of the patients were Hindu, (1498, 22.98\%) patients were Muslim and only one (0.01\%) patient was Christian. The majority of patients (4581, 70.3\%) were educated up to the high school level only and 92 (1.4\%) patients were holding graduation degrees and above. Data regarding substance abuse habits were also collected. Among the study participants, $18.3 \%$ were using tobacco or snuff and the prevalence of smoking was $2.91 \%$. A minority of $0.21 \%$ consumed alcohol on a daily basis while others had it occasionally.

NCDs are rapidly increasing at a global level and have reached epidemic proportions in many countries, largely due to globalisation, industrialisation, rapid urbanisation and lifestyle changes. ${ }^{13} \mathrm{~A}$ study in rural India during years 2010-2013 showed that NCDs accounted for 47 percent of all deaths while communicable, maternal, peri-natal and nutritional conditions all together accounted for 30 percent of the deaths. ${ }^{14}$

The disease pattern in rural India has undergone a significant shift over the last 15 years, which indicates that NCDs have become a healthcare priority. In the current study nearly 
$50 \%$ of the participants were found to be suffering from NCDs. This may be because of the fact that rural areas are equally vulnerable to non-communicable diseases due to lack of awareness, poor socio-economic status and high prevalence of smoking, tobacco chewing and alcohol consumption among rural people.

Table I.Demographic Details of Patients who visited Healthcare Mobile Clinic

\begin{tabular}{|c|c|c|}
\hline \multirow{2}{*}{ Variable } & \multicolumn{2}{|c|}{ Total patients ( $\mathbf{n}$ 6516) } \\
\cline { 2 - 3 } & Number & Percentage \\
\hline Gender & & \\
\hline Male & 3042 & 46.7 \\
\hline Female & 3474 & 53.3 \\
\hline Educational Status & & \\
\hline Illiterate & 1246 & 19.2 \\
\hline Upto High School & 4581 & 70.3 \\
\hline Upto Intermediate & 594 & 9.1 \\
\hline Graduate and above & 92 & 1.4 \\
\hline Addictions & & \\
\hline Smoking & 190 & 2.91 \\
\hline Tobacco or snuff & 1195 & 18.3 \\
\hline Alcohol & 14 & 0.21 \\
\hline Religion & & \\
\hline Hindu & 5017 & 76.99 \\
\hline Muslim & 1498 & 22.98 \\
\hline Christian & 01 & 0.01 \\
\hline
\end{tabular}

Low consumption of fruits and vegetables could probably due to the lack of awareness, and poverty especially in rural areas. WHO attributes approximately three million deaths a year from non-communicable diseases to inadequate consumption of a healthy diet. Consumption of adequate fruits and vegetables not only prevents nutrient deficiency disorders but also reduces the risk of cardiovascular diseases. In a study, it was shown that increased consumption of fruits and vegetables is associated with a $16 \%$ lower risk of cardiovascular diseases. ${ }^{6}$ At least $80 \%$ of heart diseases, strokes, and type- 2 diabetes cases and $40 \%$ of cancer cases could be avoided through a healthy diet, regular physical activity, and avoidance of tobacco-use. ${ }^{7,8}$

Disease wise summary of persons having these NCDs is given in Table 2. Generally, it is assumed that non-communicable diseases are not prevalent among as they work hard for their daily needs but above data showed that $49.82 \%$ of the village population was suffering from non-communicable diseases. It has been seen that the rural population is also susceptible to NCDs. Figure 1 (a) \& (b) shows the genderwise distribution of these diseases.

The time to take a serious note of this new health challenge in rural India has come. It is necessary to stop urbanisation of lifestyle and ensure modification of lifestyle among rural people that they can keep themselves healthy and diseases free. Unhealthy practices of tobacco chewing, smoking and alcohol consumption must be discouraged and a high intake of fruits and vegetables should be encouraged. A healthy lifestyle can be achieved by living in a healthy environment, adopting good hygiene practices, eating nutritious food, getting adequate sleep, doing regular exercise, and ensuring absence of addiction. These NCDs should be tackled by physicians with expertise, therefore the best way to treat NCDs at the village level is to provide the people access to physicians.

Table 2.Percentage of Non- Communicable Diseases (NCDs) as per the Gender of Subjects

\begin{tabular}{|c|c|c|c|c|}
\hline Diseases & Disease Code & Male (\%) & Female (\%) & Total (\%) \\
\hline Common NCDs & & & & \\
\hline Diabetes Mellitus & G-2 & 0.21 & 0.16 & 0.38 \\
\hline Hypertension & E-38 & 0.96 & 1.04 & 2.01 \\
\hline Palpitation & E-2 & 0.27 & 0.79 & 1.07 \\
\hline Bronchial asthma & D-4 & 1.41 & 0.46 & 1.87 \\
\hline Backache & L-1 & 1.19 & 2.83 & 4.03 \\
\hline Polyarthritis & L-4 & 9.65 & 14.67 & 24.32 \\
\hline Sciatica & L-8 & 0.26 & 0.62 & 0.89 \\
\hline Others & & & & \\
\hline Migraine & A-2 & 0.06 & 0.04 & 0.10 \\
\hline Vertigo and giddiness & A-25 & 0.18 & 0.21 & 0.39 \\
\hline Headache & A-36 & 1.18 & 2.14 & 3.33 \\
\hline
\end{tabular}




\begin{tabular}{|c|c|c|c|c|}
\hline Numbness & A-42 & 0.64 & 1.24 & 2.19 \\
\hline Flatulence & F-26 & 4.83 & 1.91 & 6.75 \\
\hline Hyperacidity & F-43 & 0.09 & 0.18 & 0.27 \\
\hline Anaemia & F-68 & 0.21 & 0.65 & 0.08 \\
\hline Hemorrhoid & F-96 & 0.87 & 1.15 & 2.02 \\
\hline Constipation & F-94 & 1.13 & 0.38 & 1.51 \\
\hline Malnutrition & M-36 & 0.18 & 0.44 & 0.62 \\
\hline Total & & & & 49.82 \\
\hline
\end{tabular}

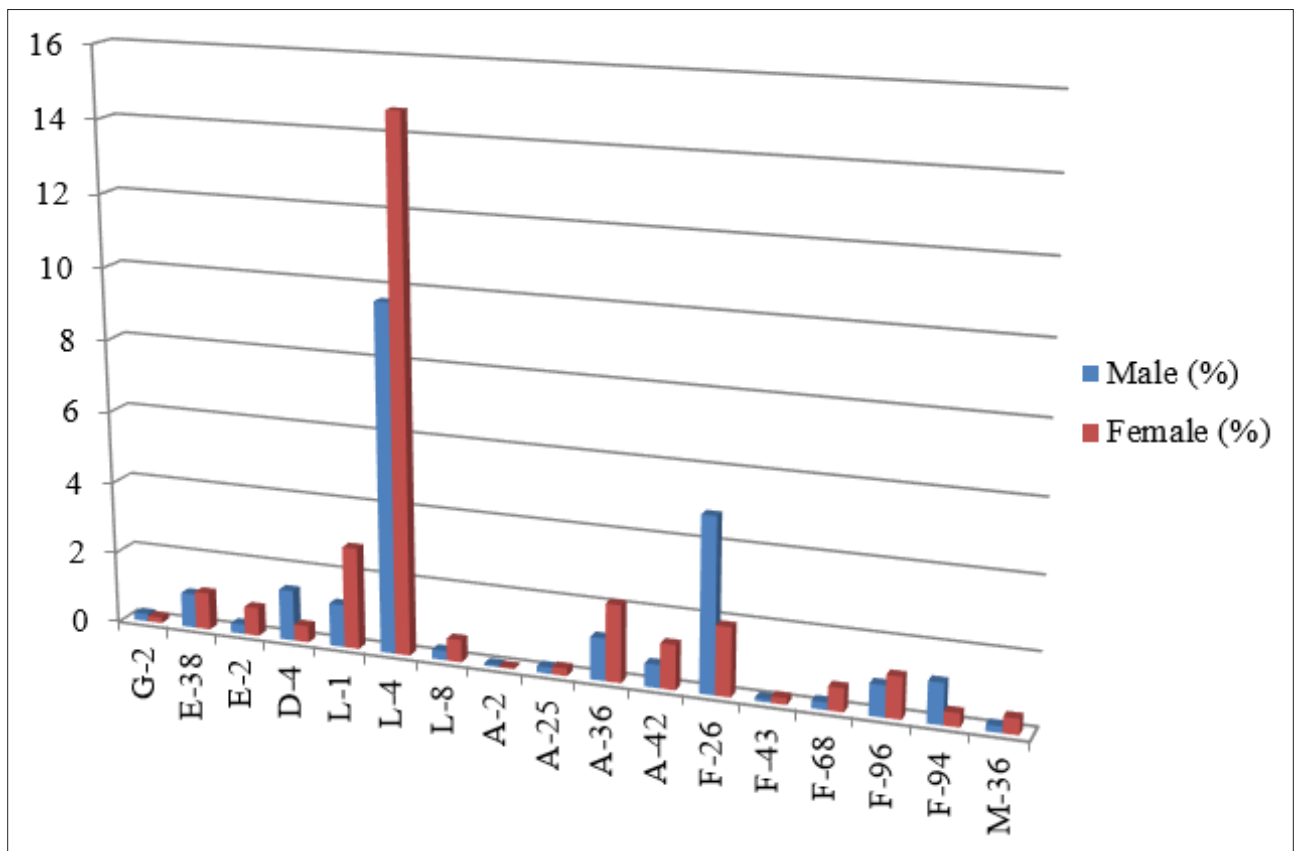

Figure I(a).Gender wise Percentage Distribution of Non-Communicable Diseases (NCDs)

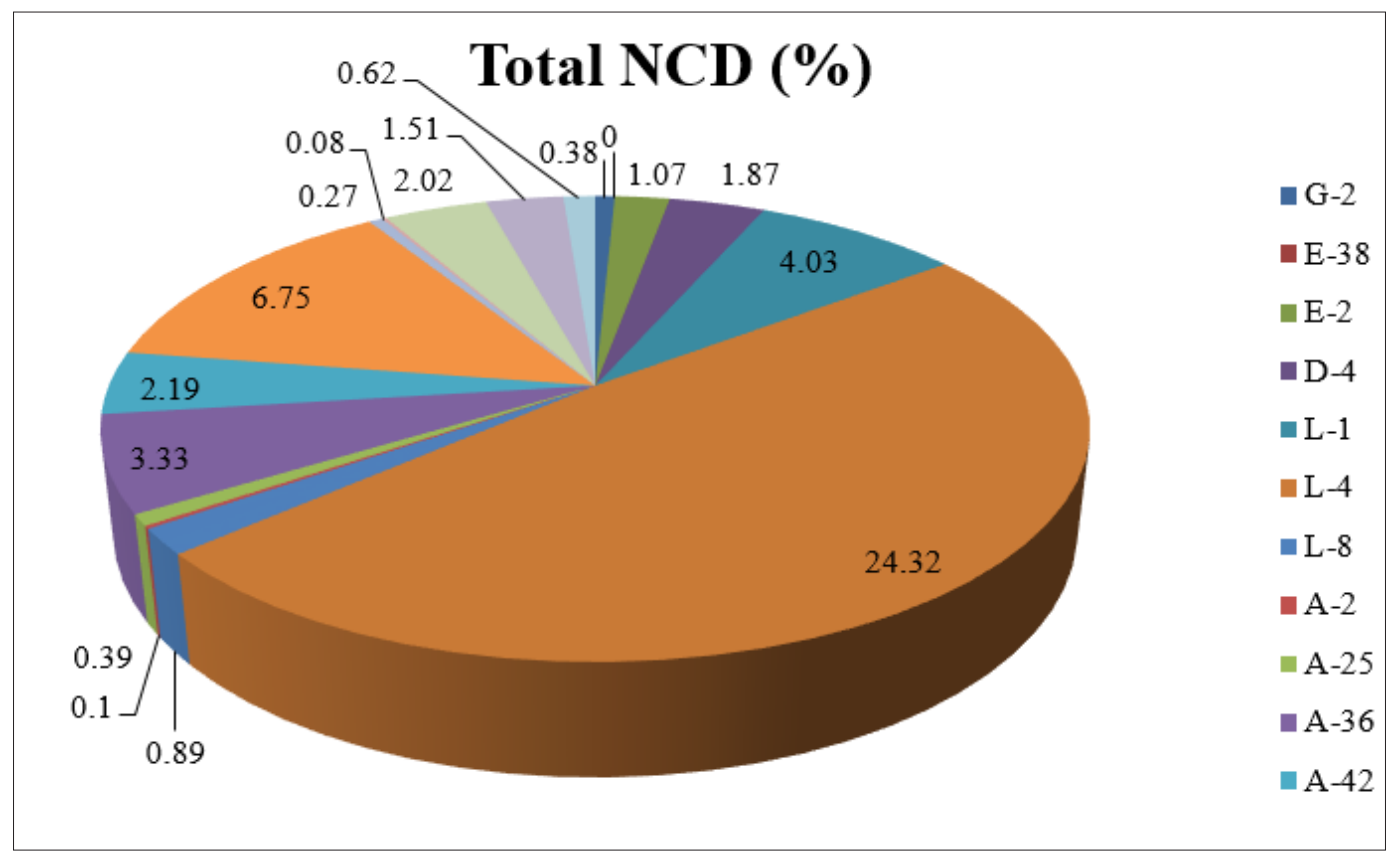

Figure I(b). Total Percentage Distribution of Non-Communicable Diseases (NCDs) 


\section{Conclusion}

Data which were collected from SCSP mobile health care OPD at clinical research unit Bhopal showed that the people who Were lived in rural areas were equally vulnerable to NCDs (since $49.82 \%$ of the subjects were suffering from NCDs) due to their lack of awareness, poor socio-economic status and also because of high prevalence of smoking, tobacco chewing, and alcohol consumption among rural men.

\section{Source of Funding}

Central Council for Research in Unani Medicine, Ministry of Ayush, Govt. of India, New Delhi.

\section{Conflict of Interest: None}

\section{References}

1. World Health Organisation. [Internet]. Noncommnunicable diseases: The socioeconomic impact of NCDs; 2018 [cited 2020 Dec 16]. Available from: https://www.who.int/news-room/fact-sheets/ detail/noncommunicable-diseases\#: :text=The\%20 rapid\%20rise\%20in\%20NCDs,costs\%20associated\%20 with\%20health\%20care

2. World Health Organisation. [Internet]. Noncommnunicable diseases: Overview; [cited 2020 Dec 16]. Available from: https://www.who.int/healthtopics/noncommunicable-diseases\#tab=tab_1

3. World Health Organisation. [Internet]. Noncommnunicable diseases; [cited 2020 Dec 16. Available from: https://www.who.int/data/gho/data/ themes/noncommunicable-diseases

4. Mohan V, Deepa R. Risk factors for coronary artery disease in Indians. J Assoc Physician Ind. 2004;52:95. [Google Scholar]

5. Reddy KS. Prevention and Control of Non-communicable Diseases: Status and Strategies. New Delhi: Indian Council for Research on International Economic Relations; Jul 2003. [Google Scholar]

6. Goyal A, Yusuf S. The burden of cardiovascular disease in the Indian subcontinent. Indian J Med Res. 2006 Sep;124:235. [PubMed] [Google Scholar]

7. Epping-Jordan JE, Galea G, Tukuitonga C, Beaglehole R. Preventing chronic diseases: taking stepwise action. Lancet. 2005 Nov;366(9497). [PubMed] [Google Scholar]

8. Aroor B, Trivedi A, Jain S. Prevalence of risk factors of non-communicable diseases in a district of Gujarat, India. J Health Popul Nutr. 2013 Mar;31(1). [PubMed] [Google Scholar]

9. Beaglehole R, Yach D. Globalisation and the prevention and control of non-communicable disease: the neglected chronic diseases of adults. Lancet. 2003 Sep;362(9387). [PubMed] [Google Scholar]
10. Yach D, Leeder SR, Bell J, Kistnasamy B. Global chronic diseases. Science. 2005 Jan;307(5708). [Google Scholar]

11. World Health Organization. The world health report: 2003: shaping the future. Report No.: ISSN 1020-3311. Geneva: World Health Organization; 2003. [Google Scholar]

12. Siegel KR, Patel SA, Ali MK. Non-communicable diseases in South Asia: contemporary perspectives. Br Med Bull. 2014 Sep;111(1). [PubMed] [Google Scholar]

13. National Health Portal. [Internet]. Non-communicable Diseases;2019 [cited 2020 Dec 16]. Available from: https://www.nhp.gov.in/healthlyliving/ncd2019

14. Examrace. [Internet]. Steps to tackle NCDs: increase in deaths due to non-communicable diseases; 2018 [cited 2020 Dec 16]. Available from: https://www. examrace.com/Current-Affairs/NEWS-Steps-to-TackleNCDs-Increase-in-Deaths-Due-to-Non-CommunicableDiseases-Important.htm 